Check for updates

Cite this: RSC Adv., 2018, 8, 27988

\title{
Formation of nanopores in DiynePC-DPPC complex lipid bilayers triggered by on-demand photo-polymerization $\uparrow$
}

\author{
Min Jung Chun, $\ddagger^{a}$ Yeol Kyo Choi $\left(\mathbb{D} \ddagger^{\mathrm{a}}\right.$ and Dong June Ahn (D) *ab
}

Vesicles have unique characteristics that enable the release of drugs as well as encapsulation while maintaining biocompatibility. A photo-polymerizable liposome composed of 1,2-bis(10,12tricosadiynoyl)-sn-glycero-3-phosphocholine (23:2 DiynePC) has been investigated as vehicles for triggered delivery of drugs to cells. In this study, we confirmed for the first time that supported lipid bilayers (SLBs) prepared with a 1,2-dipalmitoyl-sn-glycero-3-phosphocholine (DPPC)/DiynePC mixture generated pores ca. 100-300 $\mathrm{nm}$ in size on the membrane after UV polymerization. This direct observation was done by analyzing the SLBs formed with the DPPC/DiynePC mixture by employing atomic force microscopy (AFM) in a liquid environment. However, photo-polymerization did not occur in the 1,2-dioleoyl-sn-glycero-3-phosphocholine (DOPC)/DiynePC mixed bilayer and pores were not formed. A theoretical study was performed to explore the phase behavior of the lipid mixtures. A coarsegrained model of DiynePC was developed that is comparable with the Martini force field; the parameters were validated against atomistic simulations. Transition from fluidic to gel phase was observed only when DiynePC was mixed with DPPC, whereas the DOPC mixture remained fluidic over the entire domain. This implies a correlation between the formation of DiynePC-rich gel phase domains and the generation of pores after polymerization. The size of the pores were found to be controlled by the amount of polymerizable lipid which results in higher release rate of encapsulated calcein from the vesicles with larger pores.

Received 8th June 2018

Accepted 20th July 2018

DOI: $10.1039 / c 8 r a 04908 d$

rsc.li/rsc-advances polymerization, ${ }^{5}$ photo-sensitization by membrane anchored hydrophobic moieties, ${ }^{6,7}$ photo-isomerization ${ }^{8}$ and photooxidation. ${ }^{9}$ Thereby, photo-triggered liposome systems have been extensively studied. ${ }^{10-13}$ 1,2-bis(10,12-tricosadiynoyl)-snglycero-3-phosphocholine (DiynePC), one of the photopolymerizable synthetic phospholipids, has been studied as an artificial membrane component along with its unique colored feature due to the conjugated nature of the cross-linked diacetylene groups. ${ }^{14,15}$ Benefits of using polymerizable lipid in vesicle systems have been focused in order to improve physical stability. ${ }^{11,16,17}$ The concept of polymerizable lipids as phototriggered drug carriers has only recently been used for drug delivery applications. ${ }^{18,19}$ These studies have shown that DiynePC could become a good candidate for drug release owing to the fact that the vesicles containing DiynePC are biocompatible and can release the drug on demand. However, the generation of membrane pores by photopolymerization has purely been a hypothesis without evidence. Hence, in this study, we attempt to discover empirical evidence of the appearance of the pores generated upon photo-polymerization of the lipid membrane containing DiynePC, for the first time. The bilayers of 1,2dipalmitoyl-sn-glycero-3-phosphocholine (DPPC) and 1,2-dioleoyl-sn-glycero-3-phosphocholine (DOPC) mixed with DiynePC were analyzed utilizing atomic force microscopy (AFM) in liquid 
environment. Possibility of pore size control was also investigated. To gain insight at the atomistic scale, we performed molecular dynamics (MD) simulation of the DiynePC membrane mixed with different matrix lipids. The use of coarse-grained (CG) MD simulation enables examination of processes occurring at longer time scales, such as membrane domain formation, protein aggregation and structural packing properties. $^{20,21}$ The phase transition from fluidic to gel phase and polymerization-induced pore generation in the supported DPPC bilayers containing pore-opening diacetylenic lipid are discussed.

\section{Results and discussion}

\section{Morphology and size analysis}

To characterize shape and size of DPPC/DiynePC and DOPC/ DiynePC mixed vesicles, we carried out analyses with scanning electron microscope (SEM) and electrophoretic light

(a)

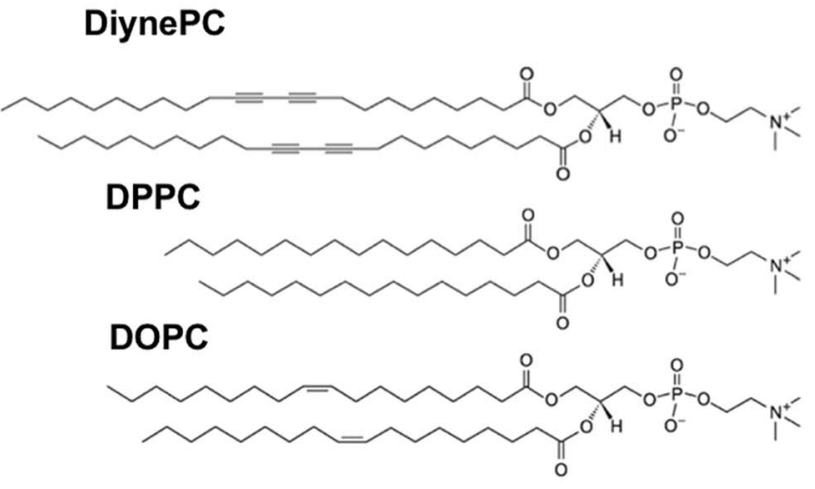

(c) DPPC/DiynePC (2:1)
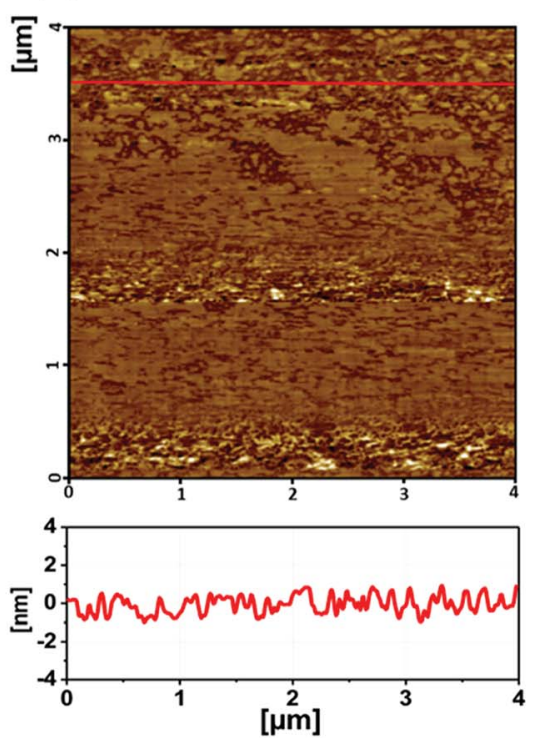

(d) DPPC/DiynePC (4:1)
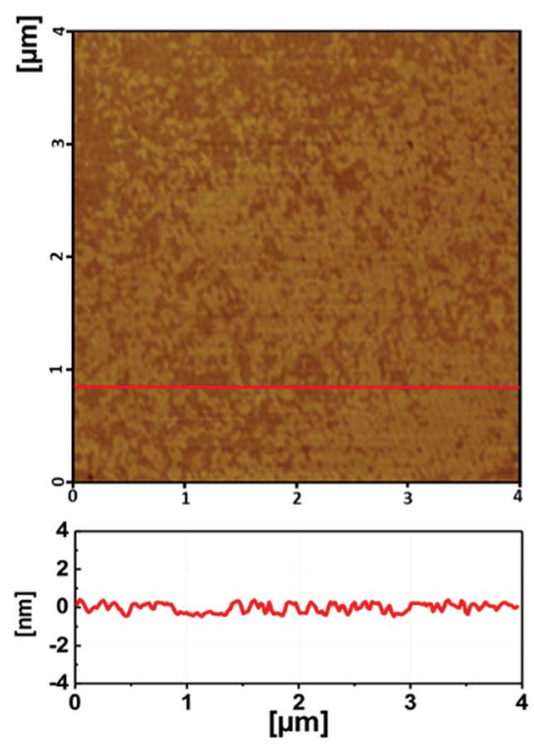

scattering (ELS). Chemical structures of the used lipid materials are shown in Fig. 1a. DPPC/DiynePC vesicles had a spherical structure; the mean vesicle diameter was $178.6 \pm 82.1 \mathrm{~nm}$. DOPC/DiynePC vesicles also had spherical structure and the mean vesicles diameter was $120.2 \pm 87.5 \mathrm{~nm}$ (ESI Fig. S1†).

\section{Formation of supported lipid bilayers}

In order to analyze the structural properties of lipid domain, SLBs of the DPPC/DiynePC and DOPC/DiynePC mixtures were fabricated on the mica substrate. Prior to structural analysis, SLB formation was validated using QCM-D measurement shown in Fig. 1b. Lipid vesicles were adsorbed on the substrate as intact vesicle state. Frequency change $(\Delta F)$ has a negative correlation with mass change on the sensor. We observed decreased $\Delta F$ due to adsorption represented by black line. Increasing dissipation change $(\Delta D)$ implies increased rigidity of material adsorbed on substrate. $\Delta D$ was increased because (b)

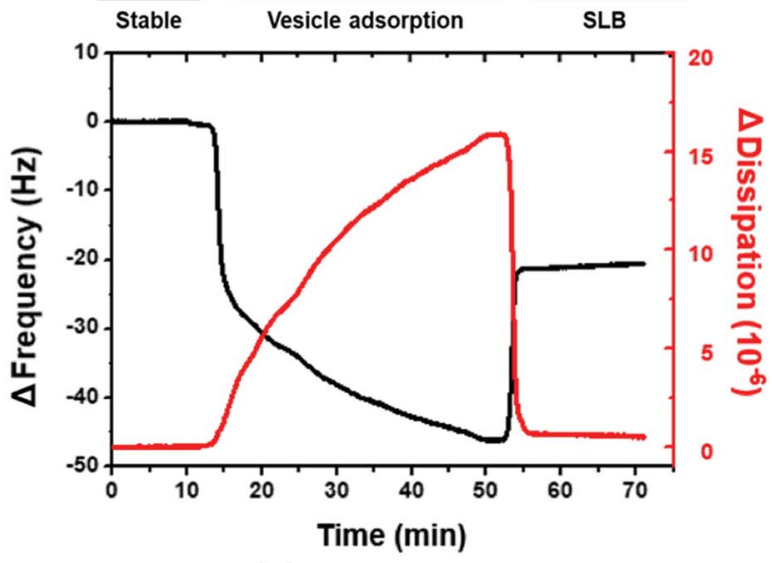

(e) DOPC/DiynePC (2:1)
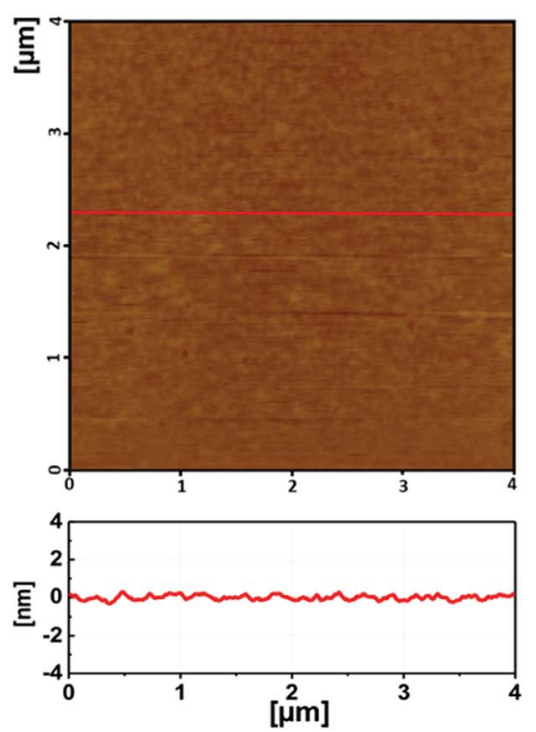

Fig. 1 (a) Chemical structure of DiynePC, DPPC and DOPC, and (b) QCM-D frequency (black line) and dissipation (red line) change as a function of time for DPPC/DiynePC vesicles on silicon dioxide coated quartz sensor. (c-e) Liquid-phase AFM images of SLBs of (b) DPPC/DiynePC (2 : 1) mixture, (c) DPPC/DiynePC (4:1) mixture and (d) DPPC/DOPC (2:1) mixture, before polymerization. 
intact vesicles had water molecules inside and this was represented by red line. $\Delta F$ was increased again, whereas $\Delta D$ was decreased sharply at $53 \mathrm{~min}$. This result indicates lipid bilayer formation caused by vesicle fusion; the mass absorbed on the sensor and the rigidity decreased as the water inside the vesicle escaped.

Fig. 1c-e are AFM images of the lipid bilayer containing DiynePC formed on mica substrate. Each figure is in sequence DPPC/DiynePC (2:1), DPPC/DiynePC $(4: 1)$ and DOPC/ DiynePC (2:1) mixed lipid bilayer, before UV irradiation. We could observe that all SLBs were fabricated on the substrate and formed lipid domains have a height difference of approximately $1 \mathrm{~nm}$. DOPC/DiynePC (2:1) bilayer had most flat surface compared with DPPC/DiynePC mixed bilayers.

\section{Simulation results}

To investigate phase behaviors of DiynePC depending on matrix lipid in atomistic scale, we first validated our CG model of DiynePC by comparison with all-atom simulation results. The parameterization of the CG model of DiynePC was based on the results from AA simulation results. The mapping scheme results of parameterization and CG simulation setup were summarized in Fig. S2, Tables S1 and S2, $\uparrow$ respectively. Fig. 2 shows snapshots of the AA and CG models of the DiynePC bilayer. The equilibrium
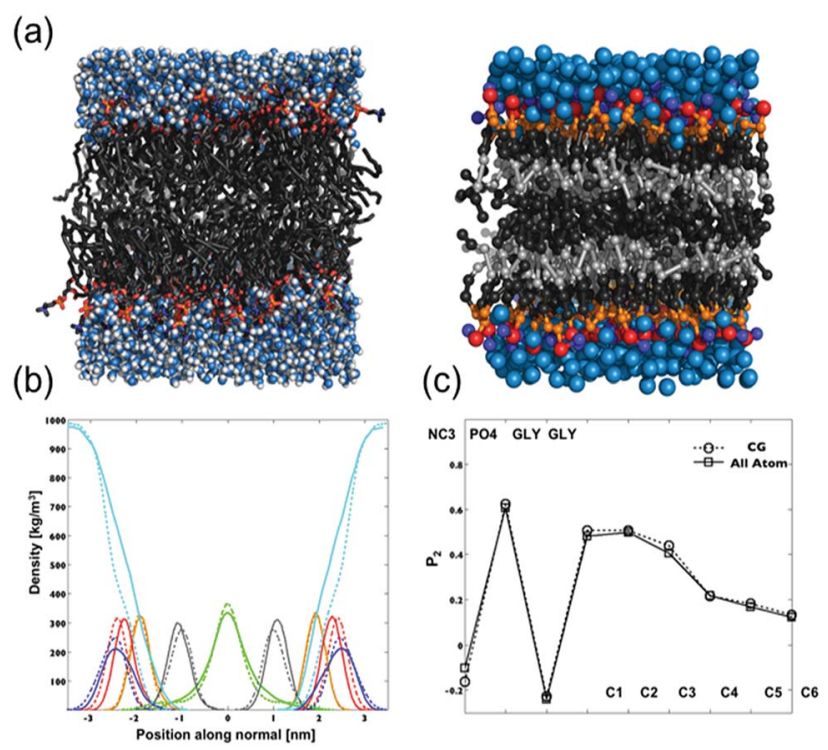

Fig. 2 (a) Snapshots of a DiynePC bilayer simulated with an atomistic model (left) and with the CG model (right). The choline groups are colored blue, phosphatidylcholine groups in red, the glycerol backbone in orange, the tails in green. A darker shade of gray is used to distinguish the terminal methyl group. Water is colored cyan. (b) Comparison of density distributions obtained from atomistic (solid lines) and coarse grained (dashed) simulations. The water density is shown in cyan, the density of NC3 groups in blue, PO4 groups in red, the glycerol backbone in orange, the terminal tail groups in green and diacetylene groups in gray. The bilayer center is located at $0 \mathrm{~nm}$. (c) P2 order parameter of consecutive bonds with respect to the bilayer normal. Results obtained for the CG bilayer are marked with circles, those for the atomistic bilayer with squares. Data are averaged over both tails. area per lipid for DiynePC in the CG model at $323 \mathrm{~K}$ is $0.61 \mathrm{~nm}^{2}$. This perfectly matches the results from AA, $0.61 \mathrm{~nm}^{2}$. Fig. $2 \mathrm{~b}$ compares the density profiles of an equilibrated CG DiynePC bilayer to profiles of one simulated in AA. Both density distributions were obtained from 128 DiynePC lipids at full saturation (50 waters per lipid). The peaks in the density distributions coincide to within $0.1 \mathrm{~nm}$, for each of the membrane components. The thicknesses of the bilayers in the AA and CG model, measured from the peaks of the phosphate distribution, were $4.6 \mathrm{~nm}$ and $4.8 \mathrm{~nm}$, respectively. We analyzed the order parameters for both the CG and AA DiynePC bilayers. As shown in Fig. 2c, the profiles were quite close. This indicated that not only the overall structure but also the alignment of the alkyl chain is well represented in the CG model of the DiynePC bilayer. The close correspondence of structural features between the bilayer of the CG and AA indicates developed CG model of DiynePC was valid to further investigation of mixture system. After parameterization of DiynePC in the CG model, we analyzed the phase behaviors of the DPPC/DiynePC and DOPC/DiynePC mixtures with a $2: 1$ molar ratio at $305 \mathrm{~K}$ to investigate the effect of the matrix lipid on the phase transition of DiynePC. Fig. 3a and $\mathrm{b}$ show the difference of the gel (or crystalline) and liquid phases when DPPC and DOPC were each mixed with DiynePC at a molar ratio of $2: 1$. Fig. 3c-e are CG configurations of lipid bilayer and height of each bilayer obtained from density distribution profiles. In the case of DPPC/DiynePC mixtures, we identified that a local hexagonal structure is seen and non-ideal mixing of the two components is visible. Position A is gel phase and this area is shown in Fig. 3c while outside position B is fluidic shown in Fig. 3d. Each domain has height about $5.42 \mathrm{~nm}$ and $4.47 \mathrm{~nm}$ respectively and this height difference around $1 \mathrm{~nm}$ can be seen (a)

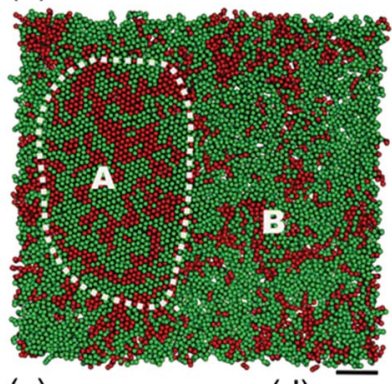

(c)

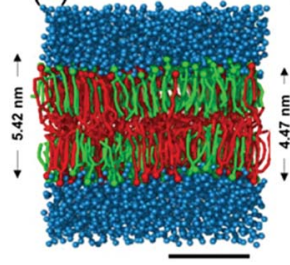

(d)

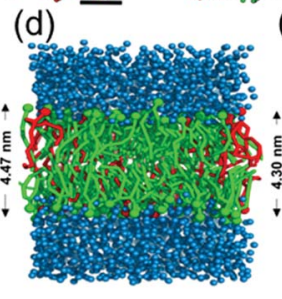

(b)

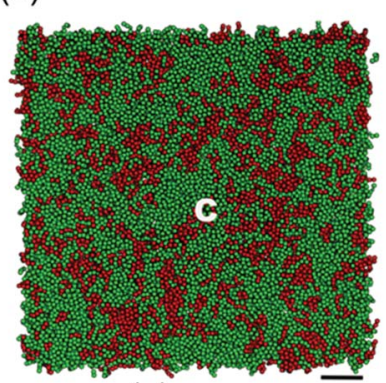

(e)

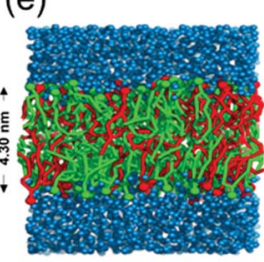

Fig. 3 Final configuration of the (a) DPPC/DiynePC and (b) DOPC/ DiynePC mixtures with molar ratio $2: 1$ at $305 \mathrm{~K}$, respectively. Water has been removed for clarity, DPPC and DOPC are shown in green, and DiynePC in red (scale bar: $3 \mathrm{~nm}$ ). Dotted lined area in (a) indicates the gel phase and the outside is fluidic. Entire area in (b) is fluidic. (c-e) Side views and bilayer thickness in (c) DPPC/DiynePC (2:1) mixed bilayer at position A, (d) DPPC-rich bilayer at position B and (e) DOPC/ DiynePC (2:1) mixed bilayer at position C. 
(a)

(b)
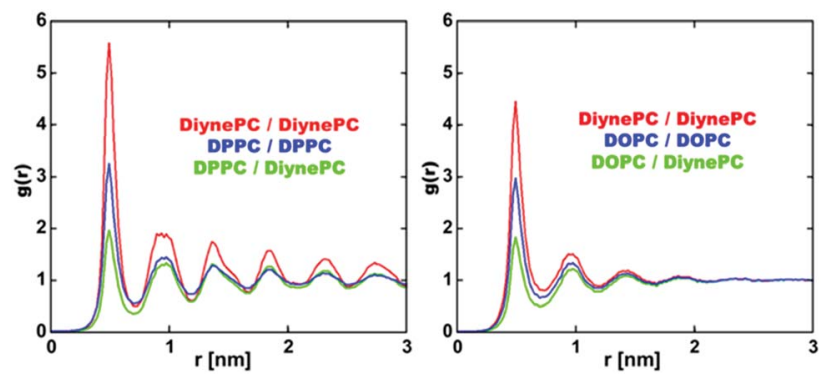

Fig. 4 Radial distribution functions (RDFs) of glycerol centers of the lipids for the (a) DPPC/DiynePC (2:1) mixture and (b) DOPC/DiynePC $(2: 1)$ mixture; probability of occurrence of pair forms in DiynePC/ DiynePC, matrix lipid/matrix lipid, and matrix lipid/DiynePC drawn compared to random mixing having RDF of 1.

in previous AFM images (Fig. 1c and d). However, the bilayer composed DOPC/DiynePC shows fluidic phase area and height about $4.30 \mathrm{~nm}$ (Fig. 3b and e). Position $\mathrm{C}$ is fluidic phase throughout all region so that the DOPC/DiynePC bilayer had much flatter surface corresponding with AFM analysis.

To investigate the phase of the DPPC/DiynePC and DOPC/ DiynePC lipid mixtures, we analyzed the radial distribution function (RDF) for both cases (Fig. 4). Glycerol beads are used to calculate RDF because the same number of them is found in all lipid components. The data show that there is a tendency toward nonideal mixing between the matrix lipid and DiynePC regardless of the matrix lipid. However, when the matrix lipid is DOPC, the $g(r)$ value converges to 1 ; it oscillates in the case of DPPC, indicating that the gel and liquid phases are formed when the matrix lipids are DPPC and DOPC, respectively. We observed that there is a different trend in the RDF analysis with different leaflets (ESI Fig. S3 $†$ ). In the case of the DOPC/DiynePC mixture, there was no significant correlation between interleaflets. However, in the DPPC/DiynePC mixture, there was a peak at $r=0$ in the DPPC/DiynePC distribution, demonstrating that it is more likely that the opposite lipid type is below the given lipid.

\section{(a) DPPC/DiynePC (2:1)}
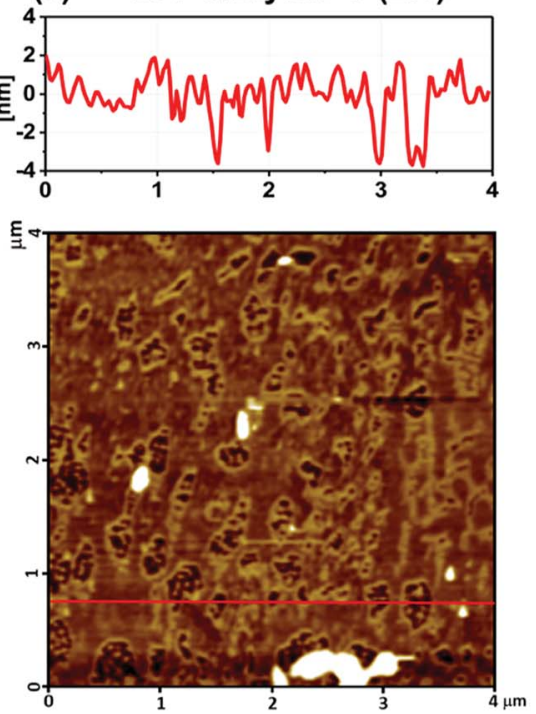

(d)

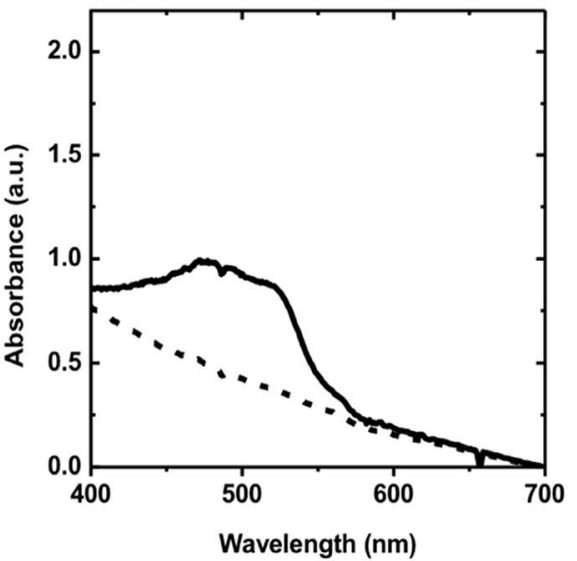

(b) DPPC/DiynePC (4:1)
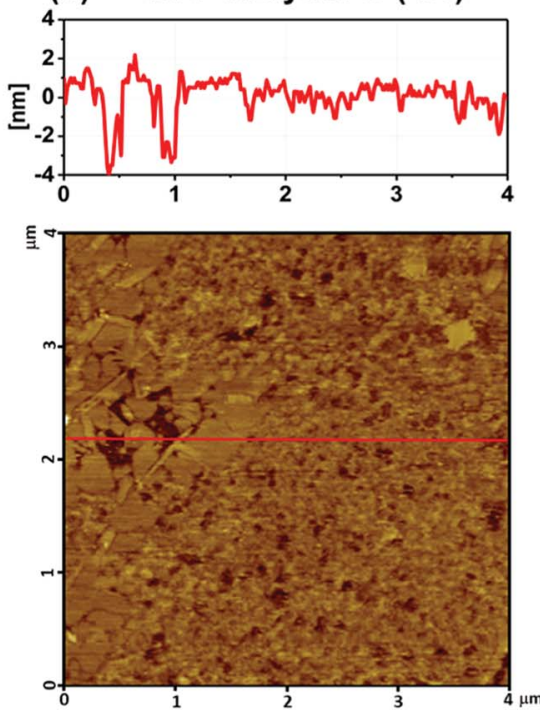

(e)

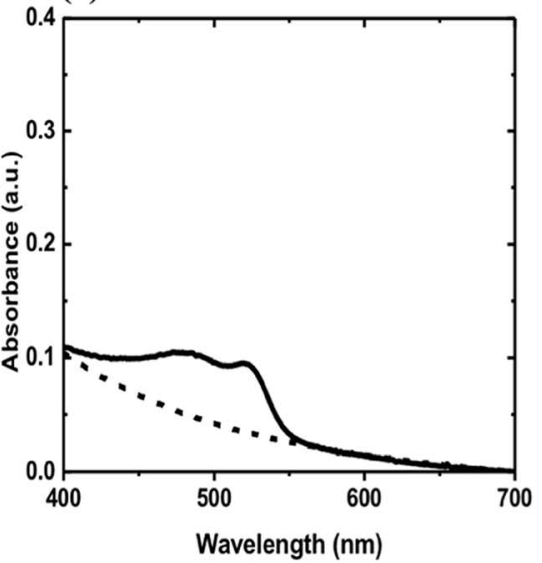

(c) DOPC/DiynePC (2:1)
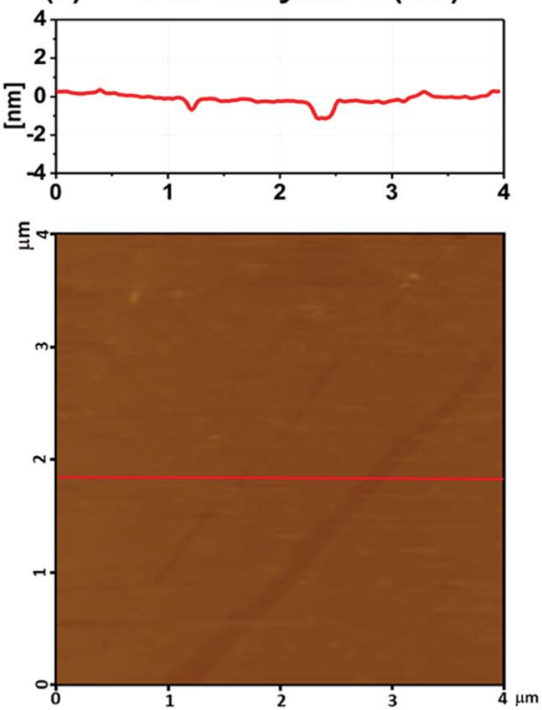

(f)

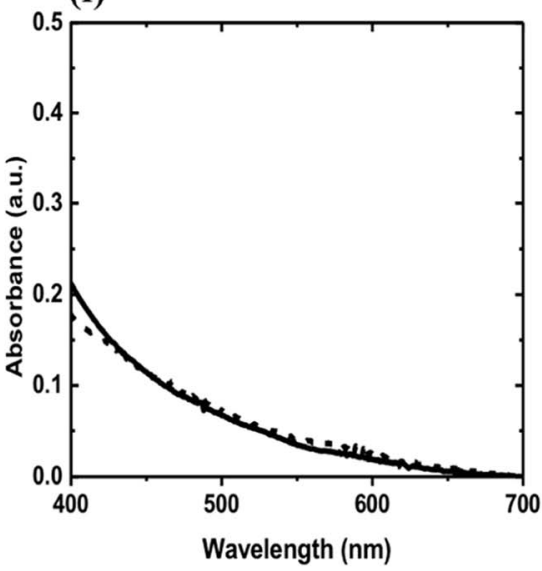

Fig. 5 (a-c) AFM images of lipid bilayers containing DiynePC after photo-polymerization (254 nm, $\left.2 \mathrm{~mW} \mathrm{~cm}{ }^{-2}, 15 \mathrm{~min}\right)$ in liquid environment. Pore size of (a) is $191 \pm 128 \mathrm{~nm}$, (b) is $122 \pm 70 \mathrm{~nm}$ and (c) is not found. (d and e) Visible absorption spectra before (dashed line) and after (solid line) polymerization of vesicles; (a and d) DPPC/DiynePC (2:1), (b and e) DPPC/DiynePC (4:1) and (c and f) DOPC/DiynePC (2 : 1) mixtures. 
Pore formation on SLBs after photo-polymerization and its size control

To observe the effect of photo-polymerization on structural change, we analyze SLBs of DPPC/DiynePC and DOPC/DiynePC mixture using AFM in liquid environment. When DPPC/ DiynePC $(2: 1)$ mixed bilayer was irradiated with UV light $\left(254 \mathrm{~nm}, 2 \mathrm{~mW} \mathrm{~cm} \mathrm{~cm}^{-2}\right.$ ) for $15 \mathrm{~min}$, pores with a height of approximately $5 \mathrm{~nm}$, the height of the bilayer, were created throughout the bilayer area (Fig. 5a). As a result of pore analysis in the membrane, the mean diameter of the pores was $191 \pm$ $128 \mathrm{~nm}$, and their total area was $2.335 \mu^{2}$. As shown in Fig. 4b, DPPC/DiynePC $(4: 1)$ mixture shows generally smaller pores than $(2: 1)$ mixture upon UV irradiation. The mean length of the pores was $122 \pm 70 \mathrm{~nm}$, and total area was $1.293 \mu \mathrm{m}^{2}$. Clearly, the mean size and total area of the pores both decreased when compared with the case of $2: 1$ mixed bilayer. Histogram analysis of pore size reveals widespread distribution in DPPC/ DiynePC (2:1) mixed bilayer (ESI Fig. S4†). On the other hand, we could not observe pores at all in DOPC/DiynePC $(2: 1)$ mixed bilayer, implying that polymerization did not occur in mixture (Fig. 5c). This result corresponds with CG simulation result explaining about formation of gel phase in DPPC/ DiynePC mixture and fluidic phase in DOPC/DiynePC mixture. Polymerization upon UV irradiation could be confirmed not only in SLBs but also in vesicle form. In the visible absorption spectra of the DPPC/DiynePC $(2: 1)$ and $(4: 1)$ vesicles, the monomeric phase did not have a specific absorption spectrum. However absorption peaks at $480 \mathrm{~nm}$ and $530 \mathrm{~nm}$ were observed after UV irradiation (Fig. $5 \mathrm{~d}$ and e). This indicates that both DPPC/DiynePC mixtures (i.e. $2: 1$ and $4: 1$ cases) were polymerized under the influence of the diacetylenic group. This could be also confirmed by naked-eye observable colorimetric change from colorless to orange-red. However, as shown in Fig. 5f, no spectral absorption was observed in the vesicle solution composed of DOPC matrix, which elucidated that polymerization of diacetylenic lipid did not occur.

\section{Effect of DiynePC composition on calcein release induced by} polymerization

From previous results, we demonstrate pore formation in DPPC/DiynePC bilayers and its size control by manipulating the composition of polymerizable lipid. We further examined the effect of controlled pore size on the release rate of encapsulated molecules. We utilized calcein, which is known as selfquenched dye, as encapsulated molecules. Photo-triggered pore formation caused self-quenched calcein to release from the DPPC/DiynePC vesicles, and fluorescence intensity increased upon UV irradiation. A total (100\%) release of calcein was obtained after the addition of Triton X-100, which is known to break the structure. As shown in Fig. 6, release rate of calcein in DPPC/DiynePC $(2: 1)$ mixed vesicles at the early stage is higher than in DPPC/DiynePC (4:1) vesicles. These results are consistent with pore size analysis describing that larger pores were formed after photo-polymerization when more poreopening diacetylenic lipid was present. In both cases, calcein
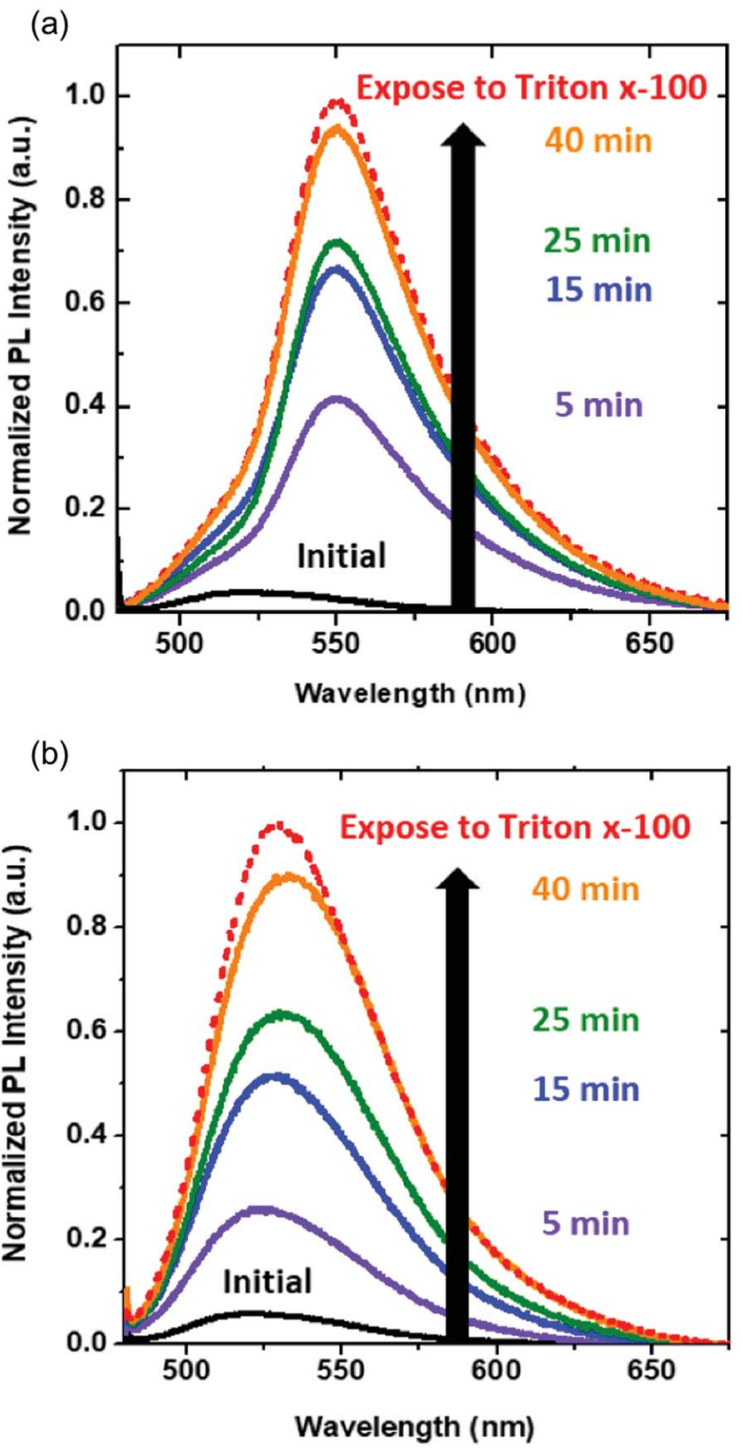

Fig. 6 Effect of composition of DiynePC on calcein release from vesicles by exposure to UV light with time. Triton X-100 line means $100 \%$ calcein release; (a) DPPC/DiynePC (2: 1) vesicles and (b) DPPC/ DiynePC (4:1) vesicles.

release continued and approached toward $100 \%$ as exposed to Triton X-100.

\section{Conclusions}

In the study described above, we confirmed photo-triggered pore generation on planar lipid bilayers containing the polymerizable lipid. Polymerization in DiynePC domains depends on which matrix lipid is used. To understand phase behaviors of lipid mixture, we carried out MD simulation of matrix/ polymerizable lipids. The results of simulation provide information that DPPC/DiynePC mixture only shows formation of the local domains which are gel phase and rich in DiynePC content. These domains are prone to photo-polymerization generating pores that is in good agreement with the observation obtained by AFM analysis. The size of pores depended 
upon the composition of DiynePC; the higher the content of polymerizable lipid, the larger the pore. In addition, this can also correlate to the release of encapsulated calcein through the vesicles' bilayers. The release rate of the calcein at the early stage was higher in DPPC containing large amount of DiynePC contents functioning as pore-opening tool. With combination of insights provided from the experiment and the theoretical studies, the pore-controlled bilayer system activated by light would be valuable for controlled drug release.

\section{Experimental methods}

\section{Preparation of polymerizable vesicles}

DiynePC, DPPC, DOPC were purchased from Avanti Polar Lipids, Inc. (Alabaster, AL, USA). The fluorescent dye calcein was obtained from Tokyo Chemical Industry. Other solvents and buffers were reagent grade. DPPC (or DOPC) and DiynePC were dissolved in chloroform $\left(1 \mathrm{mg} \mathrm{ml}^{-1}\right)$ and mixture was dried with a gentle stream of nitrogen gas to leave a thin layer on the flask. The flask was placed in a vacuum desiccator to ensure no chloroform remained. After several hours, the dried lipid was rehydrated in phosphate buffered saline (PBS buffer, $\mathrm{pH} 7.4$ ) to a final concentration of $2 \mathrm{mM}$. The solution was heated at $60{ }^{\circ} \mathrm{C}$ for $30 \mathrm{~min}$ and was subjected to five freeze-thaw cycles. The final step to make unilamellar liposomes was the extrusion method, which easily and rapidly generates uniform populations of liposomes. All equipment for extrusion was purchased from Avanti Polar Lipids, Inc. Each $1 \mathrm{ml}$ of solution was passed through the membrane 21 times using the extruder, and the finished sample was stored at $4{ }^{\circ} \mathrm{C}$. We wrapped the vesicles with aluminum foil to protect them from prepolymerization.

\section{Formation of supported lipid bilayers}

We prepared clean and flat mica discs (Ted Pella, Inc.) by continuous exfoliation using sticky tape. We fabricated SLBs by the vesicle fusion method, the most widely used, because it utilizes pure phospholipid vesicles and promotes the selfassembled bilayer spontaneously without the need for additional components. ${ }^{22,23}$ Vesicles mixed with deionized water were incubated on mica substrate at $60{ }^{\circ} \mathrm{C}$ for $90 \mathrm{~min}$. After incubation, the sample was washed gently with PBS buffer and water to remove excess residue.

\section{Fabrication of calcein encapsulated vesicles}

Calcein was used as an encapsulated fluorescent dye because it has no permeability in the membrane and self-quenching properties at concentrations above $70 \mathrm{mM}$. The basic method to make the lipid thin layer was the same as that for preparing polymerizable vesicles. It was rehydrated in deionized water containing $70 \mathrm{mM}$ calcein, and the mixture was placed in a thermostat at $60{ }^{\circ} \mathrm{C}$ for $30 \mathrm{~min}$. The extrusion method could not be used because of clogging in the membrane due to the high calcein concentration. Therefore, a probe sonicator (amplitude 20\%, $15 \mathrm{~min}$ ) was used, and the sample was stabilized at $4{ }^{\circ} \mathrm{C}$. We used a dialysis method to separate free calcein and encapsulated vesicles in this experiment. The sample was inserted in a dialysis membrane (molecular weight cut-off = 3500 ) in deionized water. Free calcein exited the membrane and the bulk solution outside the membrane became a yellow-green color. The external solution was replaced at intervals of several hours until the color did not change.

\section{Characterization}

The morphology and size of the vesicles were analyzed by using SEM (S-4700, Hitachi) and ELS (ELS Z-1000, Otsuka Electronics). The optical properties were examined with UV-Vis spectroscopy (8453, Agilent) and fluorescence spectrophotometry (F-7000, Hitachi). Bilayer formation on mica of the vesicles was validated using QCM-D (Q-Sense E4). A quartz sensor coated with silicon dioxide, a major component of mica, ${ }^{24}$ was used for the measurement (QSX-303). Based on the QCM-D analysis using $\mathrm{SiO}_{2}$ substrate for 70 min, we fabricated SLBs of DiynePC/matrix lipid mixtures on mica during same duration and these were used for further analysis. Structure analysis was carried by AFM (NX10, Park Systems) with BL-AC40RS-C2 cantilevers (spring constant with $0.09 \mathrm{~N} \mathrm{~m}^{-1}$ and resonance frequency with 110 $\mathrm{kHz}$ ) in AC mode. Measurements of all samples were carried out in liquid environment. Pore sizes in the AFM images were analyzed using the XEI program (Park Systems).

\section{Simulation methods}

All molecular dynamics runs were performed with Gromacs 4.6.7 (ref. 25-27) using the Martini force field (FF) ${ }^{28-31}$ for CG-MD and the CHARMM lipid FF. ${ }^{32}$ To control the temperature, a v-rescale thermostat $^{33}$ was used. The pressure was maintained at 1 bar using the Berendsen ${ }^{34}$ and Parrinello-Rahman ${ }^{35}$ barostats for the equilibrium and production run, respectively. Neighbor lists were built using the Verlet cut-off scheme with a cut-off radius of $1.2 \mathrm{~nm}$, and updated at each step. The linear constraint solver (LINCS) algorithm was used to constrain the bond lengths. ${ }^{36,37}$ All simulations were conducted using a leap-frog integrator with time steps of 20 and 2 fs for CG-MD and AA-MD, respectively.

\section{Coarse-grained simulation}

The DiynePC molecule was described by 16 beads using the conventional Martini scheme (ESI Fig. S2a $\dagger$ ). Detailed parameters and force constants for bond lengths and angles are listed in the ESI Table S1 $\uparrow$. Initial configurations with 2700 matrix lipids (DPPC and DOPC), 1350 DiynePC lipids, and $30375 \mathrm{~W}$ beads were obtained using the "insane.py" script. ${ }^{38}$ Periodic boundary conditions were applied to the $x, y$, and $z$ directions of the simulation cells with an initial cell size of $32 \times 32 \times 10 \mathrm{~nm}^{3}$. All bilayers were equilibrated at $323 \mathrm{~K}$ for $1 \mu \mathrm{s}$, and phase behaviors were observed at $300 \mathrm{~K}$ for $4 \mu \mathrm{s}$. An NPT ensemble was used for the CG-MD simulations with semi-isotropic pressure coupling. Configurations of the CG-MD are shown in ESI Table S2. $\dagger$

\section{Atomistic simulation}

For the simulation of the bilayer system of DiynePC, the initial configuration was obtained using the packmol program. ${ }^{39}$ The 
unit cell had dimensions of $6.5 \times 6.5 \times 8 \mathrm{~nm}^{3}$, contained 128 DiynePC and 4232 water molecules, and was equilibrated for 50 ns at $323 \mathrm{~K}$. The NPT production runs were carried out for 100 ns at $323 \mathrm{~K}$. The TIP3P model was used for water and electrostatic interactions were calculated using particle mesh Ewald ${ }^{\mathbf{4 0}}$ with a cutoff of $1.2 \mathrm{~nm}$.

\section{Conflicts of interest}

There are no conflicts to declare.

\section{Acknowledgements}

This work was supported by the National Research Foundation of Korea (NRF 2017R1A2B3006770, 2017M3D1A1039421) and the Korea University Grant.

\section{References}

1 J. O. Eloy, M. C. de Souza, R. Petrilli, J. P. A. Barcellos, R. J. Lee and J. M. Marchetti, Colloids Surf., B, 2014, 123, 345-363.

2 Q. G. Xu, Y. Tanaka and J. T. Czernuszka, Biomaterials, 2007, 28, 2687-2694.

3 D. Needham and M. W. Dewhirst, Adv. Drug Delivery Rev., 2001, 53, 285-305.

4 A. Yavlovich, B. Smith, K. Gupta, R. Blumenthal and A. Puri, Mol. Membr. Biol., 2010, 27, 364-381.

5 S. L. Regen, A. Singh, G. Oehme and M. Singh, Biochem. Biophys. Res. Commun., 1981, 101, 131-136.

6 B. Chandra, R. Subramaniam, S. Mallik and D. K. Srivastava, Org. Biomol. Chem., 2006, 4, 1730-1740.

7 A. Lavi, H. Weitman, R. T. Holmes, K. M. Smith and B. Ehrenberg, Biophys. J., 2002, 82, 2101-2110.

8 C. G. Morgan, R. H. Bisby, S. A. Johnson and A. C. Mitchell, FEBS Lett., 1995, 375, 113-116.

9 P. Shum, J. M. Kim and D. H. Thompson, Adv. Drug Delivery Rev., 2001, 53, 273-284.

10 A. Mueller and D. F. O'Brien, Chem. Rev., 2002, 102, 727-757.

11 H. Ringsdorf, B. Schlarb and J. Venzmer, Angew. Chem., Int. Ed. Engl., 1988, 27, 113-158.

12 W. Meier, Chem. Soc. Rev., 2000, 29, 295-303.

13 K.-W. Kim, H. Choi, G. S. Lee, D. J. Ahn, M.-K. Oh and J.-M. Kim, Macromol. Res., 2006, 14, 483-485.

14 G. S. Lee, S. J. Hyun and D. J. Ahn, Macromol. Res., 2018, 1-5.

15 E.-K. Ji, D. J. Ahn and J.-M. Kim, Bull. Korean Chem. Soc., 2003, 24, 667-670.

16 D. S. Johnston, L. R. McLean, M. A. Whittam, A. D. Clark and D. Chapman, Biochemistry, 1983, 22, 3194-3202.

17 J. Leaver, A. Alonso, A. A. Durrani and D. Chapman, Biochim. Biophys. Acta, Biomembr., 1983, 732, 210-218.
18 A. Yavlovich, A. Singh, S. Tarasov, J. Capala, R. Blumenthal and A. Puri, J. Therm. Anal. Calorim., 2009, 98, 97-104.

19 A. Yavlovich, A. Singh, R. Blumenthal and A. Puri, Biochim. Biophys. Acta, Biomembr., 2011, 1808, 117-126.

20 R. Faller and S. J. Marrink, Langmuir, 2004, 20, 7686-7693.

21 Y. K. Choi, D. Lee, S. Y. Lee, T. J. Shin, J. Park and D. J. Ahn, Macromolecules, 2017, 50, 6935-6944.

22 S. R. Tabaei, J. H. Choi, G. H. Zan, V. P. Zhdanov and N. J. Cho, Langmuir, 2014, 30, 10363-10373.

23 R. S. Tu and M. Tirrell, Adv. Drug Delivery Rev., 2004, 56, 1537-1563.

24 M. V. Maslova, L. G. Gerasimova and W. Forsling, Colloid J., 2004, 66, 322-328.

25 E. Lindahl, B. Hess and D. van der Spoel, J. Mol. Model., 2001, 7, 306-317.

26 D. Van der Spoel, E. Lindahl, B. Hess, G. Groenhof, A. E. Mark and H. J. C. Berendsen, J. Comput. Chem., 2005, 26, 1701-1718.

27 B. Hess, C. Kutzner, D. van der Spoel and E. Lindahl, J. Chem. Theory Comput., 2008, 4, 435-447.

28 H. Lee, A. H. de Vries, S. J. Marrink and R. W. Pastor, J. Phys. Chem. B, 2009, 113, 13186-13194.

29 S. J. Marrink, H. J. Risselada, S. Yefimov, D. P. Tieleman and A. H. de Vries, J. Phys. Chem. B, 2007, 111, 7812-7824.

30 G. Rossi, L. Monticelli, S. R. Puisto, I. Vattulainen and T. AlaNissila, Soft Matter, 2011, 7, 698-708.

31 J. J. Uusitalo, H. I. Ingolfsson, P. Akhshi, D. P. Tieleman and S. J. Marrink, J. Chem. Theory Comput., 2015, 11, 3932-3945.

32 J. B. Klauda, R. M. Venable, J. A. Freites, J. W. O'Connor, D. J. Tobias, C. Mondragon-Ramirez, I. Vorobyov, A. D. MacKerell and R. W. Pastor, J. Phys. Chem. B, 2010, 114, 7830-7843.

33 G. Bussi, D. Donadio and M. Parrinello, J. Chem. Phys., 2007, 126, 014101.

34 H. J. C. Berendsen, J. P. M. Postma, W. F. Vangunsteren, A. Dinola and J. R. Haak, J. Chem. Phys., 1984, 81, 3684-3690.

35 M. Parrinello and A. Rahman, J. Appl. Phys., 1981, 52, 71827190.

36 B. Hess, H. Bekker, H. J. C. Berendsen and J. Fraaije, J. Comput. Chem., 1997, 18, 1463-1472.

37 B. Hess, J. Chem. Theory Comput., 2008, 4, 116-122.

38 T. A. Wassenaar, H. I. Ingolfsson, R. A. Bockmann, D. P. Tieleman and S. J. Marrink, J. Chem. Theory Comput., 2015, 11, 2144-2155.

39 L. Martinez, R. Andrade, E. G. Birgin and J. M. Martinez, J. Comput. Chem., 2009, 30, 2157-2164.

40 T. Darden, D. York and L. Pedersen, J. Chem. Phys., 1993, 98, 10089-10092. 